dysarthria but no dysphasia. Vision and extraocular movements were normal. The corneal reflex was diminished on the left. There was mild weakness of the left lower facial muscles. Taste was preserved. The uvula deviated to the left and the gag reflex was diminished on the right. The tongue had no wasting but deviated to the right upon protrusion. There was no evidence of Horner's syndrome. There was pronator drift of the left upper extremity and mild weakness of the left leg. A left hemisensory deficit for pinprick, touch and proprioception involved the face, arm, leg and trunk. Muscle stretch reflexes were more active on the left and plantar responses were flexor. There was no ataxia.

Initial CT scan of the head was normal. At this point the diagnosis of medullary infarction on the right was considered but the left lower facial weakness and absent left corneal reflex could not be explained. A repeat CT scan of the head four days later revealed a right parietal infarction. Duplex ultrasound of the carotids revealed an occlusion of the distal right internal carotid artery. Magnetic resonance imaging ten days after presentation demonstrated dramatic enlargement of the diameter of the right internal carotid artery, beginning several centimeters above the bifurcation and extending to the base of the skull (fig). The signal was compatible with intramural haemorrhage. There was no flow void signal. Tapering occlusion of the right internal carotid artery was demonstrated by angiography. Heparin was started and then changed to warfarin. One year later the patient has improved ability to swallow but is otherwise unchanged. Repeat duplex ultrasound has shown no evidence of recanalisation.

Hypoglossal palsy in association with internal carotid artery dissection has been reported infrequently, occurring in $5^{\circ}$ of cases. ${ }^{2}$ The occurrence of both tenth and twelfth nerve palsies in this situation is rare. ${ }^{2}$ Hypoglossal paralysis with contralateral motor and sensory symptoms simulating a crossed hemiplegia has been reported only once. ${ }^{3}$ That patient experienced two transient ischaemic attacks in association with a persistent hypoglossal paralysis. Our patient is unique in that the lower cranial nerve palsies began simultaneously with the contralateral motor and sensory signs and the deficits persisted. The presentation mimicked that of medullary infarction. The clues of mild left facial weakness and a diminished left corneal

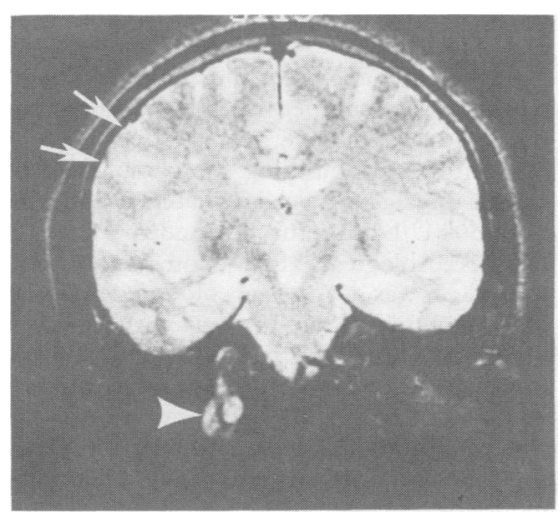

Figure Spin Echo $T_{2}$ weighted MRI (TR $2000 \mathrm{~ms}, T E=70 \mathrm{~ms}$ ) showing dissection of right internal carotid artery (arrowhead) and right parietal infarction (arrows). reflex cautioned against this localisation.

MRI showed the enlarged internal carotid artery with intramural haemorrhage and loss of the flow void signal indicating occlusion. Angiography confirmed the presence of a tapering occlusion of the internal carotid artery. The close proximity of the pharyngeal branches of the vagus, glossopharyngeal and hypoglossal nerve to the internal carotid artery in the neck makes them susceptible to compression from an expanding internal carotid artery. ${ }^{4}$ However, other factors such as ischaemia may be involved in the pathogenesis of cranial nerve palsies. MRI is superior to angiography in that the vessel wall is visualised and not just the lumen. ${ }^{5}$ Internal carotid artery dissection with ipsilateral lower cranial nerve palsies and contralateral hemiplegia should be added to the better known false localising signs. ${ }^{6}$

DAVID C HESS KAPIL D SETHI FENWICK T NICHOLS Department of Neurology, Medical College of Georgia, Augusta, Georgia, United States

1 Mokri B, Sundt TM Jr, Houser OW, Piepgras DG. Spontaneous dissection of the cervical 19:126-38.

2 Hart RG, Easton JD. Dissections of cervical and cerebral arteries. Neurologic Clinics 1983, 11:155-82

3 Goodman JM, Zink WL, Cooper DF. Hemilingual paralysis caused by spontaneous carotid artery dissection. Arch Neurol 1983;40:653-4.

4 Lieschke GJ, Davis S, Tress BM, Ebeling P. Spontaneous internal carotid artery dissection presenting as hypoglossal nerve palsy. Stroke 1988;19:1151-5.

5 Goldberg HI, Grossman RI, Gomori JM, Asbury AK, Bilaniuk LT, Zimmerman RA. Cervical internal carotid artery dissecting hemmorhage: diagnosis using MR. Radiology 1986;158:157-61.

6 Rowland LP. Tumors. In: Rowland LP, ed. Merritt's textbook of neurology, 7th ed. Philadelphia, Lea and Febiger 1984:222.

\section{Pure optic ataxia associated with a right parieto-occipital tumour}

Optic ataxia is defined as a disorder of hand movement when grasping an object in the peripheral visual field. ${ }^{1-4}$ Twenty or more cases of optic ataxia have been reported since 1967 when Garcin et al' originally introduced the idea of optic ataxia. Rondot et al, ${ }^{2}$ colleagues of Garcin, established that a disturbance of visuo-motor coordination is responsible for optic ataxia. They insisted that motor, cerebellar, and somatosensory disturbances, visuo-spatial agnosia, apraxia, and visual field defects should be excluded in diagnosing optic ataxia. However, we have found that, among reported cases, pure optic ataxia without these symptoms has been confirmed in only two cases. ${ }^{34}$ We report a case of optic ataxia in a pure form. Our case supports Rondot's concept that optic ataxia is a specific entity independent of other neurological disorders.

In November 1983 a 47 year old right handed man with no previous disease noticed difficulties in grasping objects, errors in estimating distances, and misty vision. On 23 January 1984, when these symptoms became slightly aggravated, the patient was admitted to our hospital. On admission, he was alert and well-orientated. His verbal functions and memory were normal. Visual acuity was normal in each eye. The visual field was full in

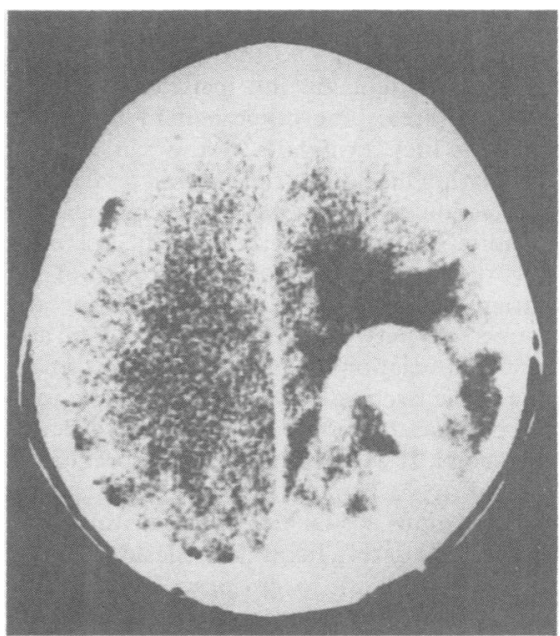
density lesion is situated in the right parietooccipital area.

each eye. External ocular movements were completely normal in every direction, and no diplopia was detected. Optic fundi were clear bilaterally. With the visual line fixed to the centre of the visual field, it was impossible for him to grasp an object at the periphery of the left homonymous visual field with the left hand, while easily possible with the right hand. Grasping an object in the right homonymous visual field with the left or right hand was performed smoothly. The patient did not exhibit visual inattention. Disturbances of the cranial nerves were not found. Muscle strength of the four extremities was symmetrically normal. Deep sensation such as joint sense, vibration sense, and deep pain was not disturbed. Two point discrimination, graphaesthesia, stereognosis, and double simultaneous stimulation were all normal. Superficial sensation was also normally preserved. There were no disorders of cerebellar functions. In the neuropsychological examination, there was no aphasia, apraxia, or visuo-spatial agnosia. On the basis of these neurological findings, it was diagnosed that the patient was suffering from optic ataxia.

Enhanced CT revealed a high density lesion in the right parieto-occipital region (fig). Subtotal removal of the tumour was performed, and it was histopathologically shown to be a malignant lymphoma. Systemic examinations revealed no malignant lymphoma elsewhere. We concluded that the tumour was a primary malignant lymphoma in the right parieto-occipital area. The residual tumour completely disappeared after whole brain irradiation of a total of $50 \mathrm{~Gy}$.

In 1967 Garcin et al reported the case of a patient who had difficulty in grasping objects at the periphery of the visual field, when the patient's visual line was fixed.' They named this neurological disorder "optic ataxia (ataxie optique)". It has been revealed on CT and necropsy that optic ataxia is caused by a lesion at the junction of the parietal and occipital lobes. ${ }^{1-4}$ Rondot et al $^{2}$ have established the concept that optic ataxia is a kind of disconnection syndrome which occurs due to interruption of nerve fibres connecting the visual association field (area 19) and the motor association field (area 6) which is responsible for identifying targets in space, for choosing a course of action, and for programming movement. ${ }^{5}$ It is thought that one half of the 
nerve fibres which originate in the visua association field go to the ipsilateral motor association field via the ipsilateral parietooccipital area. The other half of the nerve fibres, which originate in the same visual association field, go to the contralateral motor association field via the ipsilateral parietooccipital area and thereafter via the posterior part of the corpus callosum. A lesion at the parieto-occipital area might interrupt the connection between the visual association and motor association fields. If the lesion is small, one of the two pathways which go to the ipsior contralateral motor association field can be disturbed. It will be difficult therefore for the patient to grasp an object in the contralatera homonymous visual field with a unilateral, either right or left, hand. If the lesion is large, it will be difficult to grasp an object in the contralateral homonymous visual field with bilateral hands because of damage to both pathways.

Symptoms similar to optic ataxia can be seen in patients with motor disturbance, cerebellar symptoms, somatosensory disorders, visuo-spatial agnosia, apraxia, or visual field defects. Rondot et al maintained that these symptoms should be excluded in diagnosing optic ataxia, but most of the reported cases of optic ataxia have had some of these other symptoms. ${ }^{124}$

Our report on the existence of pure optic ataxia, also reported by Piccirilli et al, ${ }^{3}$ Hirose et $a l,{ }^{4}$ implies that optic ataxia can exist as a symptom independent of those symptoms we describe previously. Our CT findings and those by Hirose et al revealed that the lesion is located at the junction between the parietal and occipital lobes.

In Piccirilli's case, the patient had difficulty in grasping an object in his hemivisual field using either hand. Hirose et al reported a case similar to that of Piccirilli's. In our case, the patient could not grasp an object in his left homonymous visual field using his left hand. These three cases strongly support the anatomical explanation of optic ataxia offered by Rondot et al.

We conclude that optic ataxia is an important symptom which indicates the existence of a parieto-occipital lesion.

SEIICHI ANDO KOUZO MORITAKE Department of Neurosurgery, Shimane Medical University, Izumo, Japan Correspondence to: Dr Ando, Department of
Neurosurgery, Shimane Medical University, Enyacho 89-1, Izumo City, Japan.

1 Garcin R, Rondot P, Recondo J. Ataxie optique localisée au deux hémichamps visuels homonymes gauches (Etude clinique avec présentation d'un film). Rev Neurol 1967;116: 707-14.

2 Rondot P, Recondo J, Ribadeau Dumas JL. Visuomotor ataxia. Brain 1977;100:355-76.

3 Piccirilli M, Piccinin GL, Ricci S, Taramelli M, Piccolini C, Floridi P. Pure optic ataxia. Acta Neurol (Napoli) 1983;5(1):38-42.

4 Hirose G, Kawada J, Oda R, Kitagawa Y, Kosoegawa H. Visuomotor ataxia. Clinica and CT scan studies in three cases. Clin Neurol 1985;25:342-50.

5 Ghez C. Introduction to the Motor Systems. In: Kandel ER, Schwartz JH, eds. Principles of neural science, 2nd ed. New York: Elsevier, neural science,
Palatal myoclonus successfully treated with clonazepam

We have recently successfully treated a case of severe chronic palatal myoclonus.

A 72 year old right handed woman presented with vomiting, "shaking" of the tongue and jaw, a choking sensation during sleep and slurred speech. Her condition had started three years previously with vomiting which occurred mainly at night. This was initially mild and intermittent and was not related to food. She denied any nausea, abdominal pain or dysphagia. She lost $22 \mathrm{~kg}$ in three years. One year before admission she developed what she described as a constant shaking of the tongue and mouth which persisted during sleep and appeared to wake her up during the night with a choking sensation. To prevent this she slept propped up with six pillows. Six months later she developed intermittent jerky movements of the left arm.

She had partial bowel resection for ileocaecal tuberculosis many years before. She had no relevant family or social history and she was taking paracetamol and lorazepam 2.5 mgs at night. She had not received treatment with neuroleptic drugs.

On examination she had continuous rapid rhythmical involuntary movements of the lips, soft palate and tongue. These were not affected by voluntary action and persisted during sleep. Occasionally she also had brief jerky movements of the left hand. The rest of the neurological and general physical examination was normal.

Her full blood count, urea and electrolytes, liver function tests and brain computerised tomography scan were normal. Barium studies of the upper gastro-intestinal tract were also normal.

A diagnosis of palatal myoclonus was made and treatment with clonazepam $0.5 \mathrm{mgs}$ three times a day was started. One week later all her symptoms resolved completely. Palatal myoclonus is a rare condition characterised by rhythmical involuntary contractions of the oro-pharangeo-palatine muscles and the diaphragm at a nystagmoid rate $(120-180 / \mathrm{min}$ ute). Persistence of these movements during sleep distinguishes palatal from other forms of myoclonus and indeed from all involuntary movements. In addition to the classical features of palatal myoclonus, our patient had vomiting which occurred mainly when lying flat and improved in the upright position. This is probably due to mechanical stimulation of the pharangeal receptors by the soft palate.

Palatal myoclonus is due to a lesion interrupting the central tegmental tract, ${ }^{1}$ the olivo-dentate pathways or the contralateral dentate nucleus ${ }^{2}$ and leading to secondary vacuolar degeneration and pseudohypertrophy of the inferior olive. These lesions are usually due to brainstem infarction or idiopathic degeneration. Other causes include tumours, head injury and rarely neurosyphilis, multiple sclerosis, syringobulbia, and amyotrophic lateral sclerosis.

Until recently there was no effective treatment for palatal myoclonus. ${ }^{34}$ Phenobarbitone and sodium valproate were tried with limited success. Reduced brain serotonin metabolites have been reported in palatal myoclonus and 5 hydroxytryptophan (in addition to carbidopa) was used successfully in the treatment of one case. ${ }^{4} \mathrm{We}$ used small doses of clonazepam, a drug which increases brain 5 hydroxytryptamine, ${ }^{5}$ with excellent response. We suggest that clonazepam is worth considering in the treatment of palatal myoclonus.

AMO BAKHEIT PO BEHAN

Glasgow University Department of Neurology, Institute of Neurological Sciences, Southern General Hospital,

Glasgow

Correspondence to: Dr AMO Bakheit.

1 Herrmann C Jr, Brown JW. Palatal myoclonus: a reappraisal. J Neurol Sci 1967;5:473-92.

2 Koeppen AH, Barron CD, Dentinger MP. Olivary hypertrophy: histochemical demonstration of hydrolytic enzymes. Neurology 1980;30:471-80.

3 Walton JN, ed. Paroxysmal and convulsive disorders. Brain diseases of the nervous system, 9th ed. Oxford: Oxford University Press, 1985.

4 Magnussen I, Dupont E, Prange-Hansen Aa, Olivarius B de Fine. Palatal myoclonus treated with 5-hydroxytryptophan and a decarboxylase inhibitor. Acta Neurol Scand 1977; lase inhibito $551-3$.

5 Chadwick D, Harris R, Jenner P, Reynolds EH, Marsden $\mathrm{CD}$. Manipulation of brain
serotonin in the treatment of myoclonus. serotonin in the treat
Lancet $1975 ; \mathrm{ii}: 343-4$

\section{Body building and rhabdomyolysis}

In 1988 we reported three cases of myoglobinuria following the first session of body building. ' Our theory was that body building might be a frequent cause of exertional muscle necrosis. Since then, four other cases of rhabdomyolysis following body building have been referred to us.

Case 1 was a 20 year old student with no history of neuromuscular diseases. The day after a 50 minute session of body building, he complained of diffuse myalgia and of passing dark urine. Four days later his serum creatine kinase (CK) was $62380 \mathrm{U} / 1$ (normal up to 170). One month after neurological examination, the serum $\mathrm{CK}$ and electromyography were normal. Open muscle biopsy, performed on the quadriceps muscle and processed as previously reported, ${ }^{1}$ did not show abnormalities.

Case 2 was a 20 year old student who regularly practised tennis, skiing, and body building. $\mathrm{He}$ interrupted his sports activities to have surgery for recurrent shoulder subluxation. Six months later it was recommended that he take up body building. The day after the first session, the serum $\mathrm{CK}$ revealed a marked increase of the enzyme $(16000 \mathrm{U} / 1)$ The patient was asymptomatic. Neurological examination was normal. Serum CK values returned to normal one week later. Electromyography and muscle biopsy performed one month later were normal.

Case 3 was a 25 year old housewife, who had practised competitive athletics between the age of 12 and 18 years. After the first session of body building she complained of diffuse myalgia. Five days later serum $C K$ was $3500 \mathrm{U} / \mathrm{l}$, but returned to normal in a few days. Neurological examination was normal.

Case 4 was a 19 year old student who complained of diffuse myalgia without myoglobinuria after the first session of body building. Three days later the serum CK was $11000 \mathrm{U} / 1$ and returned to normal in one week. Neurological examination was normal.

All four patients denied using steroid hormones or other drugs.

These four cases confirm the occurrence of rhabdomyolysis with and without myoglobinuria $^{1-3}$ after body building and suggest 